\title{
Características microestruturais de concretos adicionados de C-S-H e nanotubos de carbono
}

\author{
Microstructural characteristics of concrete added \\ with $\mathrm{C}-\mathrm{S}-\mathrm{H}$ and carbon nanotubes
}

Marcelo Adriano Duart ${ }^{1}$

Instituto Federal do Paraná, IFPR, Campus Foz do Iguaçu, Departamento de edificações, Av. Araucária, 780, Bairro Vila A, CEP: 85860-000, Foz do Iguaçu, Paraná, Brasil.

e-mail: marceloduart@yahoo.com.br

\begin{abstract}
RESUMO
A incorporação de nanomateriais é uma possibilidade para o melhoramento e modificação características ou propriedades em materiais. A utilização de nanotubos de carbono (NTC) tem sido pesquisada em concretos para aumento da resistência à tração, à compressão, módulo de elasticidade entre outras características como redução de permeabilidade. Buscou-se melhorar a cinética de hidratação dos silicatos do cimento pela criação de espaços de nucleação e crescimento de estruturas cristalinas tornando a microestrutura mais densa, conforme verificados pela análise microscópica e porosimetria. Corpos de prova em microconcreto tiveram a resistência à tração testadas aos 28 dias. As porcentagens de NTC $(0,0 \%$ e $0,2 \%)$ e C-S-H $(0,0 \%$ e $2,0 \%)$ foram variadas, bem como a razão $\mathrm{Ca} / \mathrm{Si}(0,8$ e 1,2) de C-S-H. No total foram produzidos 5 tipos de compósitos que tiveram suas características comparadas ao material de referência (sem adições). O compósito C2-0,8 (com $2 \%$ de $\mathrm{C}-\mathrm{S}-\mathrm{H}$ de relação $\mathrm{Ca} / \mathrm{Si}=0,8$ ) apresentou resistência à tração $18,0 \%$ maior que o material de referência $(\mathrm{R})$, já para o compósito misto CT2-0,8 (2\% C-S-H Ca/Si = 0,8 e 2\% NTC) o aumento da resistência de $33 \%$ comparado à referência. O compósito T2 (adicionado apenas $0,2 \%$ de NTC) apresentou $13 \%$ de acréscimo de resistência se comparado ao material R. O uso de NTC, em concretos ou outras misturas cimentícias, pode ser potencializado se empregado em conjunto com C-S-H (precipitado). Isto melhora a hidratação dos silicatos do cimento através da criação de espaços de nucleação que favorecem o crescimento cristalino e reduzem a porosidade conforme análise em microscopia eletrônica e testes de porosimetria por intrusão de mercúrio realizadas nas amostras.
\end{abstract}

Palavras-chave: C-S-H, Nanotubos de carbono, resistência à tração na flexão.

\section{ABSTRACT}

The incorporation of nanomaterials is a possibility for the improvement and modification of characteristics or properties in materials. The incorporation of nanomaterials is a possibility for the improvement or even the creation of some characteristics or properties in materials. The use of carbon nanotubes (CNT) has been researched in concretes to increase the compressive and flexural strength, modulus of elasticity among other characteristics such as reduced permeability. The aim was to improve the hydration kinetics of cement silicates by creating nucleation spaces and growth of crystalline structures making the microstructure denser, as verified by microscopic analysis and porosimetry. Microconcrete specimens had tensile strength tested at 28 days. The percentages of NTC $(0.0 \%$ and $0.2 \%)$ and $\mathrm{C}-\mathrm{S}-\mathrm{H}(0.0 \%$ and $2.0 \%)$ were varied, as well as the $\mathrm{Ca} / \mathrm{Si}$ ratio (0.8 and 1.2) of C-S-H. In total, 5 types of composites were produced, and their results were compared to the reference material (without additions). The composite $\mathrm{C} 2-0.8$ (with $2 \% \mathrm{C}-\mathrm{S}-\mathrm{H}$ of $\mathrm{Ca} / \mathrm{Si}=0.8$ ) presented tensile strength $18.0 \%$ higher than the reference material (R), already for the composite CT2-0, 8 ( $2 \% \mathrm{C}-\mathrm{S}-\mathrm{H} \mathrm{Ca} / \mathrm{Si}=0.8$ and $2 \% \mathrm{CNT}$ ) the increase in resistance of $33 \%$ compared to the reference. The composite $\mathrm{T} 2$ (added only $0.2 \%$ of CNT) presented $13 \%$ of increase of resistance when compared to material $\mathrm{R}$. The use of NTC, in concrete or other cementitious mixtures, can be enhanced if used in conjunction with $\mathrm{C}-\mathrm{S}-\mathrm{H}$ (precipitate). This improves the hydration of cement silicates by creating nucleation spaces that favor crystalline growth and reduce porosity as seen in electron microscopy analysis and mercury intrusion porosimetry tests performed on the samples.

Keywords: C-S-H, carbon nanotubes, flexural tensile strength. 


\section{1. INTRODUÇÃO}

Soluções baseadas em nanotecnologia são cada vez mais buscadas em pesquisas para contribuir no melhoramento das propriedades dos materiais a base de cimento Portland para compensar suas deficiências e ainda para agregar características não intrínsecas como: resistência à tração, impermeabilidade, condutividade elétrica, entre outras.

O concreto de cimento Portland é um dos materiais mais consumidos no planeta e suas aplicações ainda não foram superadas de forma efetiva na construção de edifícios, rodovias, pontes, barragens entre outras, devido a sua versatilidade e facilidade de ser moldado em variadas formas e pelas características de resistência e durabilidade que podem ser especificadas e executadas de forma racional.

Nesta pesquisa buscou-se testar da incorporação de adições de nanomateriais como partículas de Silicato de Cálcio Hidratado (C-S-H), produzidas em laboratório por precipitação e nanotubos de carbono (NTC) com objetivo de verificar os efeitos ocorridos na microestrutura de concretos produzidos, em especial com respeito a sua densidade, estrutura porosa e resistência à tração na flexão. Para tanto foram produzidos concretos com diferentes quantidades de adições de NTC e partículas de C-S-H.

A adição de nanotubos de carbono foi testada por MEDEIROS et al. [1], em argamassas. Os pesquisadores variaram os teores de adições de NTC entre 0,1 e $0,5 \%$ (em relação a massa total de cimento) e verificaram que houve crescimento da resistência à compressão axial dos corpos de prova testados, aos 28 dias. Tais autores relataram que os concretos produzidos no NTC tinham microestrutura mais compacta e que resultou entre outras características aumento da resistência à compressão axial (27\% superiores à resistência do material controle).

Os NTC formam uma rede extensiva de reforço, na matriz de materiais cimentícios, o que é benéfico para melhorar a integridade dos materiais conforme VEEDU [2]. Este pesquisador comparou o desempenho mecânico de cimento reforçado com fibras de carbono $(\mathrm{FC})$ e materiais à base de cimento reforçados com NTC e verificou que a resistência à compressão é $30 \%$ maior e quando se analisa a resistência e flexão os resultados podem ser $100 \%$ maior que o material com FC. Na análise o pesquisador atribuiu a melhora da resistência mecânica a dois fatores: reforço por material resistente a tração (NTC) e a microestrutura menos porosa.

O uso de partículas de C-S-H já foi testado com sucesso por THOMAS, JENNINGS e CHEN [3] que verificaram ser possível aumentar a nucleação e crescimento de partículas nos espaços vazios entre silicatos do cimento Portland $\left(\mathrm{C}_{3} \mathrm{~S}\right.$ e $\left.\mathrm{C}_{2} \mathrm{~S}\right)$, que normalmente seria uma região porosa.

Tais autores demonstraram que a atividade térmica foi intensificada quando se adicionava partículas de C-S-H nos materiais cimentícios. Desta forma foi confirmada o implemento de nucleação e a possibilidade de melhoramento da estrutura cristalina, pelo crescimento de cristais resistentes (C-S-H secundário).

KUNTHER, FERREIRO e SKIBSTED [4] investigaram a influência da relação Ca/Si na resistência à compressão de prismas de argamassas produzidos com diferentes estequiometrias e constataram que quanto menor a relação $\mathrm{Ca} / \mathrm{Si}$, maior a resistência à compressão e menor o volume molar. Estes pesquisadores testaram 4 diferentes relações $\mathrm{Ca} / \mathrm{Si}(0,83 ; 1,00 ; 1,25$ e 1,50). Aos 28 dias, a resistência dos prismas com relação $\mathrm{Ca} / \mathrm{Si}$ variou de aproximadamente 7,0 $\mathrm{MPa}(\mathrm{Ca} / \mathrm{Si} 1,50 \mathrm{e} \mathrm{Ca} / \mathrm{Si}$ 1,25) para aproximadamente $15,0 \mathrm{Mpa}$, para prismas produzidos com C-S-H de relação $\mathrm{Ca} / \mathrm{Si}=0,83$, ou seja, um aumento de mais de $100 \%$ de resistência, conforme ilustra a figura 1. 


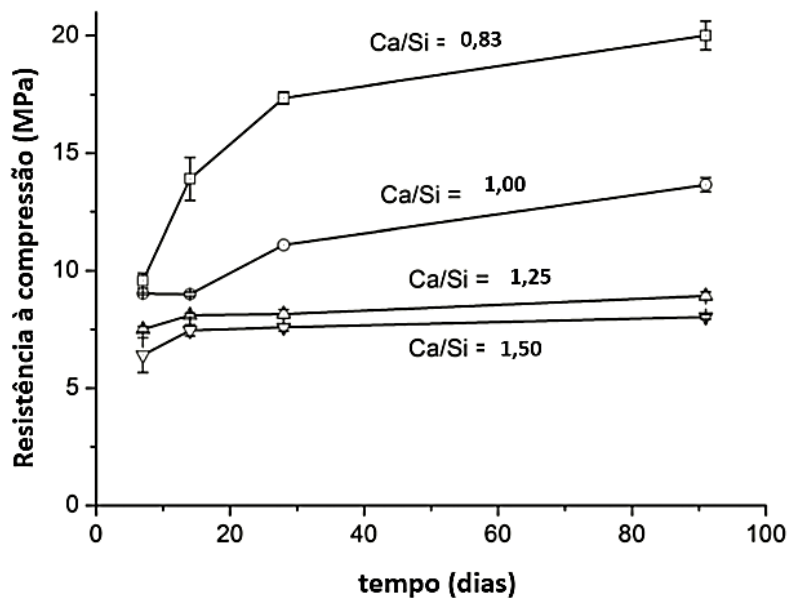

Figura 1: Resistência à compressão de prismas de argamassa, para diferentes relações $\mathrm{Ca} / \mathrm{Si}$. Fonte: Adaptado de $\mathrm{Ku}$ nther, Ferreiro e Skibsted [4].

KIRGIZ [5] testou valores para relação $\mathrm{Ca} / \mathrm{Si}$ variando de $0,53,0,63$, e 0,96 nas idades de 7, 28 e 90 dias respetivamente e concluíram que quanto menor o valor da relação $\mathrm{Ca} / \mathrm{Si}$ melhores são resultados de resistência mecânica devido a maior afinidade química do C-S-H produzido com os demais silicatos presentes no cimento Portland, desta forma as partículas de C-S-H produzido serviram de núcleo para crescimento de C-S-H secundário.

Deve-se considerar que a adição de partículas de C-S-H pode melhorar a microestrutura de materiais a base de cimento Portland reduzindo a porosidade total como também causando a redução do diâmetros dos poros (refinamento dos poros), desde que sejam produzidas adições com a relação $\mathrm{Ca} / \mathrm{Si}$ adequada, pois isto influência na sua reatividade química também na resistência dos cristais resultantes.

TAYLOR [6] fez estudos e comparou a estrutura cristalina do C-S-H com outros minerais e concluiu que a estruturas atômicas do gel C-S-H são semelhantes àquelas que ocorrem naturalmente minerais cristalinos como Tobermorita, Jennita.

Conforme TAYLOR [7] o volume ocupado por hidratos é geralmente menor do que o correspondente volume estequiométrico de cimento e água, desta forma os vazios são consequentemente formados. Portanto o cimento endurecido é um material não homogêneo e predominantemente poroso.

O C-S-H produzido em laboratório quando usado como adição tem a vantagem de ser um material da mesma origem química do C-S-H naturalmente produzido pela hidratação dos silicatos do cimento Portland (Silicato tricálcico $(\mathrm{CaO})_{3} \mathrm{SiO}_{2}$ e Silicato dicálcico $(\mathrm{CaO})_{2} \mathrm{SiO}_{2}$. DUART [8] testou o C-S-H produzido em laboratório verificou a melhora da microestrutura dos concretos o que resultou em menor porosidade e consequentemente aumento da resistência a compressão e módulo de elasticidade.

DUART [8] relata ainda que existe melhorias microestruturais na escala nanométrica uma vez que as partículas de C-S-H adicionadas atuam como espaço de nucleação e possibilitam crescimento cristalino de hidratos resultantes dos silicatos do cimento Portland.

Para KUNTHER, FERREIRO e SKIBSTED [4], nas pastas produzidas apenas por materiais a base de cimento Portland existe a permanência de grãos de clínquer que ficam inertes, desta forma não produzem materiais resistentes. Desta forma percebe-se como é importante soluções que possam propiciar processo hidratação mais efetivo. Com adições de C-S-H (produzidos no laboratório) as reações são priorizadas e restam menos materiais inertes e, portanto, a microestrutura fica mais compacta e resistente.

\section{MATERIAIS E MÉTODOS}

\subsection{Produção do C-S-H precipitado.}

Foram produzidos $\mathrm{C}-\mathrm{S}-\mathrm{H}$ com duas relações $\mathrm{Ca} / \mathrm{Si}$ diferentes $(0,8$ e 1,2$)$. O processo adotado foi precipitação direta da mistura dos reagentes: $\mathrm{Ca}\left(\mathrm{NO}_{3}\right)_{2}$ e $\mathrm{Na}_{2} \mathrm{SiO}_{3}$. A precipitação química foi estimulada através de sonicação em equipamento de ultrassom por imersão.

As quantidades de reagentes foram devidamente obtidas pelo cálculo estequiométrico realizado para garantir o controle da relação $\mathrm{Ca} / \mathrm{Si}$ conforme tabela 01 . Este detalhe foi necessário pois conforme muitos pesquisadores já verificaram, a relação $\mathrm{Ca} / \mathrm{Si}$ é determinante da reatividade do $\mathrm{C}-\mathrm{S}-\mathrm{H}$ produzido. 
Tabela 1: Quantidade de reagentes, conforme cálculo estequiométrico.

\begin{tabular}{ccc}
\hline $\mathrm{Ca} / \mathrm{Si}$ & 0,8 & 1,2 \\
\hline Reagente & $\mathrm{g}$ & $\mathrm{g}$ \\
\hline $\mathrm{Ca}\left(\mathrm{NO}_{3}\right)_{2} \cdot 4 \mathrm{H}_{2} \mathrm{O}$ & 100,00 & 100,00 \\
\hline $\mathrm{Na}_{2} \cdot \mathrm{SiO}_{3}$ & 241,83 & 161,22 \\
\hline
\end{tabular}

A técnica adotada foi a mesma usada por THOMAS, JENNINGS e CHEN [3] que se baseia na precipitação direta dos componentes citados, realizada em recipiente fechado e submetido à sonificação em banho (imersão por 60 minutos). Após a precipitação procedeu-se a filtragem em funil de Büchner, com uso de filtro de papel.

Em seguida o material foi submetido a secagem em estufa para posteriormente ser transformado em pó em moinho de bolas cerâmicas, durante 12 horas.

A presença de C-S-H foi confirmado pela difração de raio X do material produzido, figura 2 .
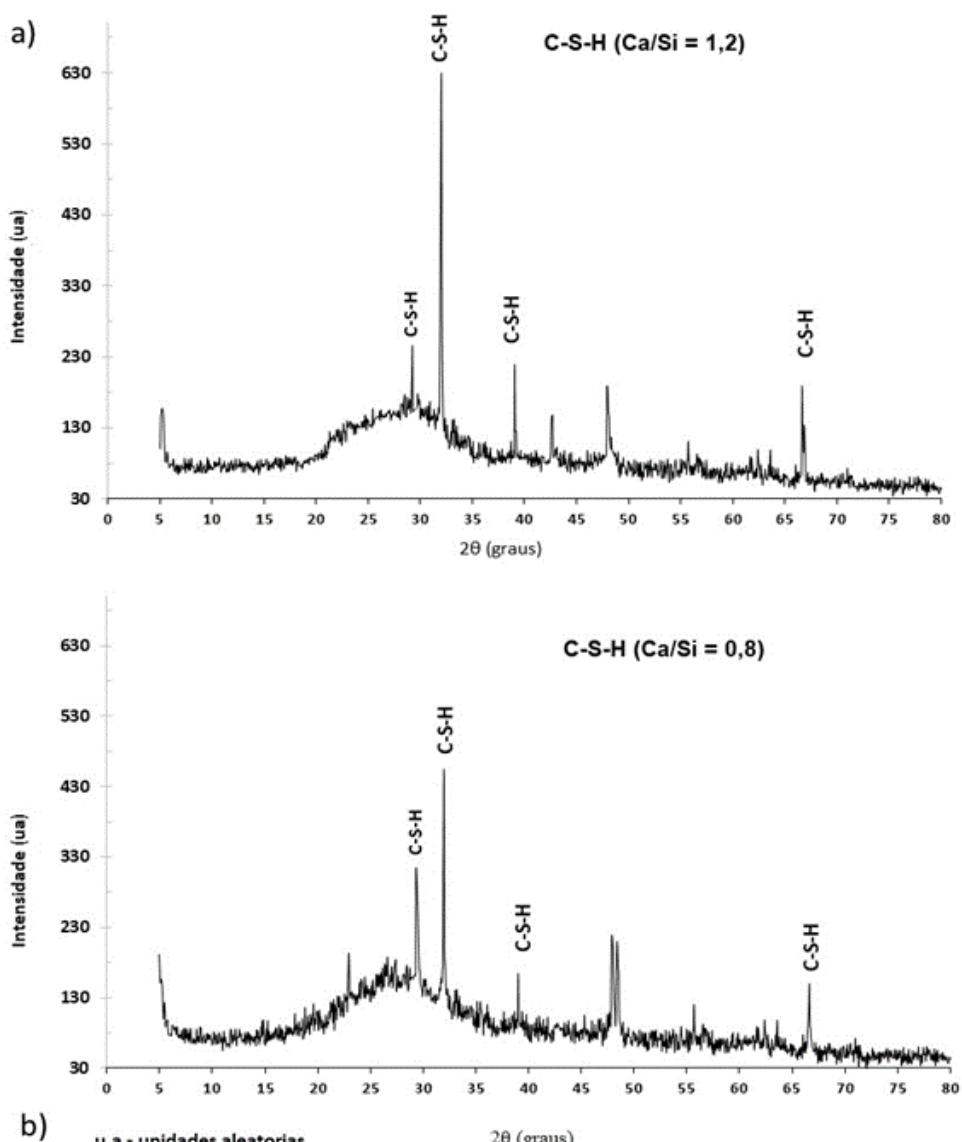

b)

Figura 2: Difração de raio X - amostras de C-S-H.

\subsection{Produção e tratamento químico dos nanotubos de carbono de paredes múltiplas (NTCPM).}

Os NTC usados foram do tipo paredes múltiplas produzidos segundo a técnica de deposição química à vapor (DQV). Basicamente os NTCPM são produzidos a partir de precursores (óxido de ferro e magnésio) que foram submetidos a fonte de carbono, o gás metano $\left(\mathrm{C}_{2} \mathrm{H}_{4}\right)$.

Foi utilizado um forno tubular programado para atingir a temperatura $850^{\circ} \mathrm{C}$ durante um fluxo de gás inerte, Argônio (Ar), no interior de um tubo de quartzo. Ao atingir a temperatura programada, foi liberado o fluxo de gás etileno $\left(\mathrm{C}_{2} \mathrm{H}_{4}\right)$, por $20 \mathrm{~min}$, na vazão ajustada de $350 \mathrm{ml} / \mathrm{min}$, que serviu como fonte de carbono. 
Para anular a hidrofobicidade os NTCPM sofreram oxidação química, por imersão em meio ácido $\left(\mathrm{H}_{2} \mathrm{SO}_{4}+\mathrm{HNO}_{3}\right.$, na proporção de 3:1). A concentração dos ácidos usados foi: $96 \%$ para $\mathrm{H}_{2} \mathrm{SO}_{4}$ e $65 \%$ para $\mathrm{HNO}_{3}$. Em seguida a solução foi e submetida sonicação (por imersão) durante 1 hora, segundo técnica proposta por MOKHTAR et al. [9]. Este processo garantiu que os NTCPM pudessem ser dispersos em água, pois como produzidos estes materiais são hidrofóbicos.

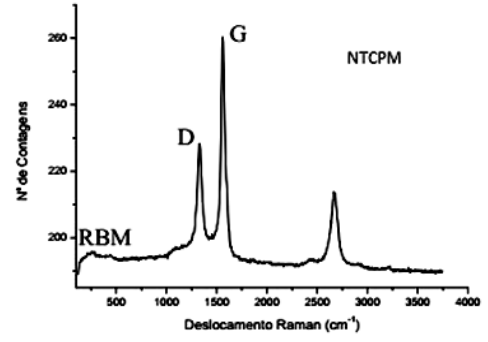

a)

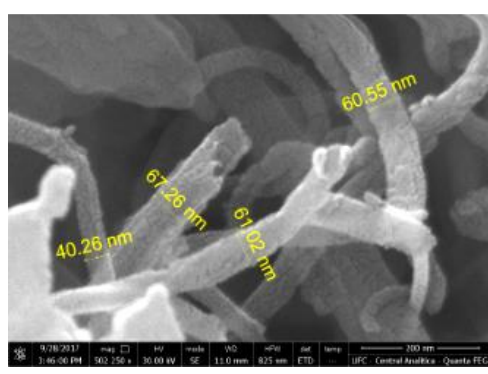

b)

Figura 3: Espectroscopia Raman e MEV da amostra de NTCPM.

Após este procedimento, a mistura foi lavada com água destilada até atingir o $\mathrm{pH}$ neutro e então realizado o processo de filtragem seguido de secagem em estufa. A análise da figura 3a, confirma o espectro típico dos NTCPM. Na análise por microscopia foi possível determinar o diâmetro médio dos nanotubos (entre 40 e $60 \mathrm{~nm}$ ).

\subsection{Produção de corpos para realização de ensaios.}

Foram produzidos 5 diferentes tipos de compósitos (em microconcreto). O concreto de referência foi produzido com os seguintes materiais: cimento Portland do tipo CPV- ARI-RS (ABNT NBR 16697:2018 [10]; Areia normal para ensaio de cimento (ABNT NBR 7214:2015 [11]) e brita 1 de origem basáltica passante na peneira de malhas $2,4 \mathrm{~mm}$. Os demais traços utilizaram os mesmos materiais, porém foram variadas as adições de nanomateriais resultando nos seguintes traços de microconcretos:

- Traço (R): referência, não possui adição alguma;

- Traço (T): adição (2\% em relação a massa de cimento) de NTCPM

- Traços (CT): adições (0,2\% em relação a massa de cimento) de NTCPM e C-S-H (Ca/Si-0,8 e $\mathrm{Ca} / \mathrm{Si}=1,2)$.

- Traços $(\mathrm{C})$ : adições (2\% em relação a massa de cimento) de $\mathrm{C}-\mathrm{S}-\mathrm{H}(\mathrm{Ca} / \mathrm{Si}-0,8$ e Ca/Si=1,2).

As quantidades de adições estão apresentadas na tabela 2.

Tabela 2: Quantidades de adições

\begin{tabular}{lccccccc}
\hline & $\mathrm{Ca} / \mathrm{Si}$ & $\mathrm{R}$ & $\mathrm{T}$ & $\mathrm{C}-0,8$ & $\mathrm{C}-1,2$ & $\mathrm{CT}-0,8$ & $\mathrm{CT}-1,2$ \\
\hline $\mathrm{C}-\mathrm{S}-\mathrm{H}$ & 0,8 & - & - & $2,0 \%$ & - & $2,0 \%$ & - \\
C-S-H & 1,2 & - & - & - & $2,0 \%$ & - & $2,0 \%$ \\
NTCPM & & - & $0,2 \%$ & - & - & $0,2 \%$ & $0,2 \%$ \\
\hline
\end{tabular}

* em relação a massa de cimento

Foram produzidos corpos de prova prismáticos para o ensaio de tração na flexão com dimensões $4 \mathrm{~cm} \mathrm{x}$ $4 \mathrm{~cm} \times 16 \mathrm{~cm}$ em formas metálicas, as mesmas usadas para ensaio de argamassa. $O$ processo de moldagem seguiu recomendações da norma ABNT NBR 13279:2005 [12]. Os ensaios de resistência mecânica foram realizados na máquina de ensaio universal, aos 28 dias.

Para a análise de porosimetria por intrusão de mercúrio (PIM) e microscopia eletrônica de varredura (MEV/FEG), foram recolhidos fragmentos dos corpos de prova ensaiados à flexão. Estes fragmentos com dimensões aproximadas de $6 \mathrm{~mm}$. Os fragmentos sofreram tratamento para paralisar as reações químicas. $\mathrm{O}$ tratamento foi realizado por imersão em álcool etílico absoluto, durante 24 horas. Posteriormente procedeu-se 
secagem, em estufa a $60{ }^{\circ} \mathrm{C}$, durante 12 horas. Após foram acondicionados em recipientes hermeticamente fechados.

A análise de PIM foi realizada no equipamento AutoPore IV 9500 V1.09 da micromeritics e análise de MEV/FEG foi realizada no equipamento microscópio eletrônico de varredura com canhão de emissão de campo, Quanta FEG 450, dotado de análise complementar por espectrômetro de energia dispersiva (EDS) e detetor X-Max de $80 \mathrm{~mm}^{2}$ de área ativa.

\section{RESULTADOS E DISCUSSÕES}

\subsection{Porosimetria por intrusão de mercúrio}

Para esta análise foi considerado a classificação da International Union of Pure and Applied Chemistry (IUPAC). Conforme IUPAC (2014) [13], os poros são classificados em:

Microporos: poros com diâmetro menores que $2 \mathrm{~nm}(\theta<2 \mathrm{~nm})$;

Mesoporos: poros com diâmetro entre $2 \mathrm{~nm}$ e $50 \mathrm{~nm}(2 \mathrm{~nm}>\theta<50 \mathrm{~nm})$;

Macroporos: poros com diâmetro maiores que $50 \mathrm{~nm}(\theta>50 \mathrm{~nm})$;

Considerando a análise de porosimetria, figura 04 , a traço sem adições (R) foi o mais poroso com porosidade total em torno de $20 \%$. Sua porosidade foi tomada como referência (100\%) conforme figura 4-a. O material $\mathrm{R}$ apresentou distribuição dos poros bem diversificada com percentual de macroporos (diâmetro > $1,0 \mu \mathrm{m}$ ) de $24 \%$ e apenas $14,2 \%$ de microporos (diâmetro $100 \leq \theta \leq 1000 \mathrm{~nm}$ ) conforme figura 4-b, demonstrando distribuição dos poros não refinada. O refinamento acontece pela redução da porosidade total e pela substituição de poros de maior diâmetro (macroporos) por poros menores (mesoporos e microporos). Desta forma a permeabilidade pode ser reduzida e proteger o material contra ataques de agentes agressivos (intemperismo, gases ácidos, cloretos entre outros) além de aumentar a resistência mecânica (compressão e tração).O material em destaque foi o CT2-0,8, traço com as adições de NTCPM e C-S-H (relação Ca/Si = 0,8) cuja porosidade total foi apenas de $11 \%$, representando apenas $55 \%$ da porosidade do traço R. A distribuição da porosidade apresentou-se refinada, com o percentual de macroporos reduzidos para 5,1\%, figura 4 - b.
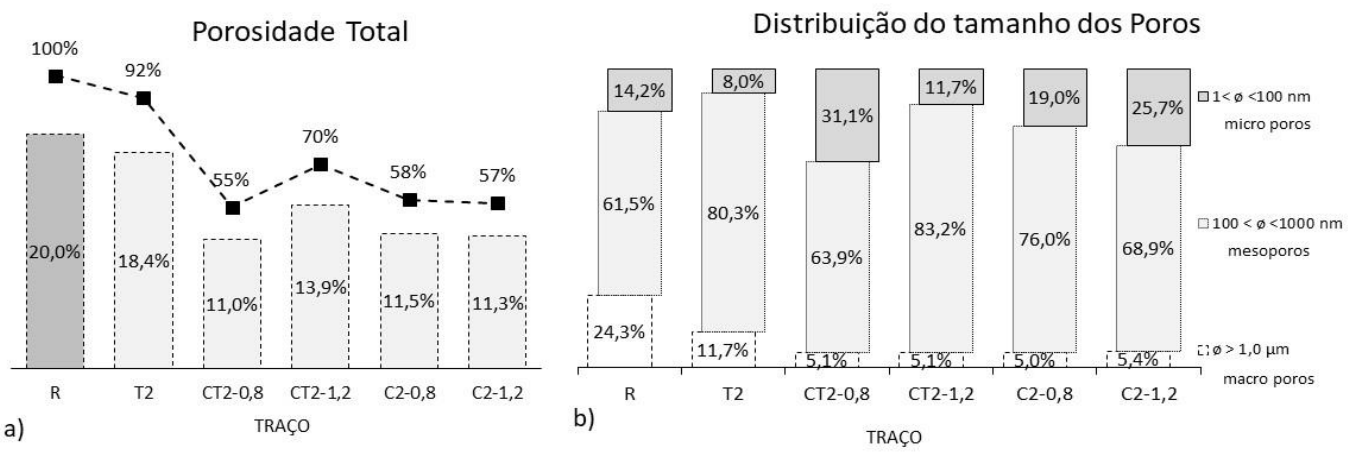

Figura 4: Porosidade total e distribuição dos tamanhos dos poros.

\subsection{Permeabilidade}

Na figura 5-a, verifica-se que o traço CT2-0,8 apresentou a menor permeabilidade e este dado está coerente com porosidade total e distribuição dos poros, figura 4, visto que a redução da porosidade e a quantidade de macroporos torna o material mais denso conforme figura 5-b, uma característica importante e desejável para aumentar a durabilidade. 
Permeabilidade (mDarcy)

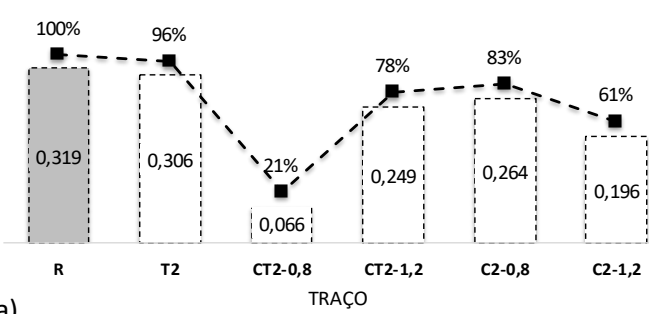

a)

Figura 5: Permeabilidade e densidade total.
Densidade total $(\mathrm{g} / \mathrm{mL})$

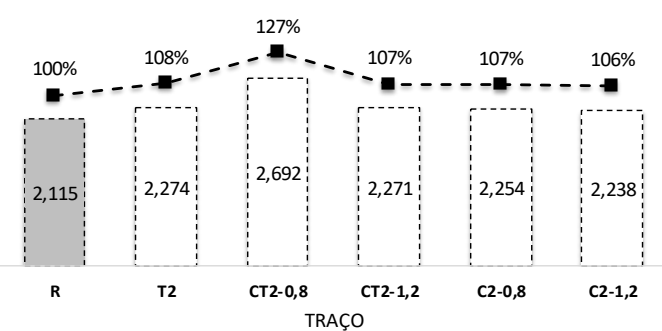

b)

\subsection{Resistência à tração}

Considerando a resistência a tração na flexão, figura 6, percebe-se que todos os traços com adições tiveram melhor desempenho que o traço R. CT2-0,8 foi o traço menos poroso, mais denso e mais resistente, seguido pelo traço CT2-1,2. Estes dois traços são compostos de adições mistas de NTCPM e C-S-H. Este comportamento deve-se pel redução da porosidade e da melhor distribuição destas. A presença das partículas de NTCPM contribuiram para aumentar a resistência à tração. A adição pura de NTCPM, traço T2, levou o aumento de resistência à tração de $13 \%$.

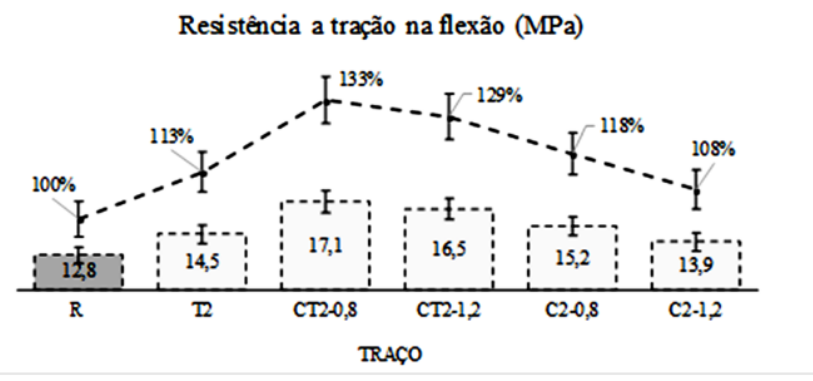

Figura 6: Resistência à tração na flexão.

EVANGELISTA et al. [14] obtiveram resultados de resistência à flexão 15\% superiores em argamassas produzidas com $0,4 \%$ de CNT, quando comparadas ao material de referência. Estes pesquisadores testaram também outras características como deformação e ductilidade e consideram que o aumento da resistência à flexão se da pelo capacidade de suportar mais carga em função do aumento da ductilidade proporcionado pelos CNT e também pelo efeito de ponte proporcionado pela presença destas nanopartículas quando bem dispersas na matriz cimentícia.

Pesquisadores que analisaram a porosidade total e densidade [14] verificaram que o uso de CNT reduz a porosidade e deixa os materiais mais densos em especial com teor de adição de $0,4 \%$, mas estes autores não aprofundaram a análise sobre a distribuição dos poros que propicia determinar a modificação microestrutural em função do refinamento dos poros e neste caso mesmo que a porosidade aumente e a densidade diminua, como foi o caso da adição de $0,6 \%$ [14], o que pode estar acontecendo é o aumento de microporos contra a redução dos macroporos.

Quando se usa adição de C-S-H combinado com NTC, traços CT2-0,8 e CT2-1,2 verifica-se que o aumento da resistência à tração foi da ordem de $30 \%$, conforme figura 6 , estes traços combinados obtiveram o melhor resultado de resistência à tração. Conforme os resultados todos os concretos com adições superaram o traço referência e as opções com adição isoladas de $\mathrm{C}-\mathrm{S}-\mathrm{H}$ em especial com relação $\mathrm{Ca} / \mathrm{Si}=0,8$ foi a de melhor resultado, sendo superada apenas pelas misturas com adições mistas.

ABDOLHOSSEINI QOMI et al. (2014) [15] pesquisaram através de simulação computacional a estrutura molecular do C-S-H para diferentes relações $\mathrm{Ca} / \mathrm{Si}$ e verificaram que dos menores para os maiores valores desta relação, a estrutura vai ficando cada vez mais amorfa. 
Estes autores verificaram ainda que para relação $\mathrm{Ca} / \mathrm{Si}=1,1$ a estrutura lamelar apresenta menores defeitos que $\mathrm{C}-\mathrm{S}-\mathrm{H}$ com relações $\mathrm{Ca} / \mathrm{Si}=1,5$ e $\mathrm{Ca} / \mathrm{Si}=1,8$. Aumentando a relação $\mathrm{Ca} / \mathrm{Si}$ pontes tetraédricas são removidas da cadeia de sílica criando estrutura mais desordenada e portando mais frágil, conforme figura 7.

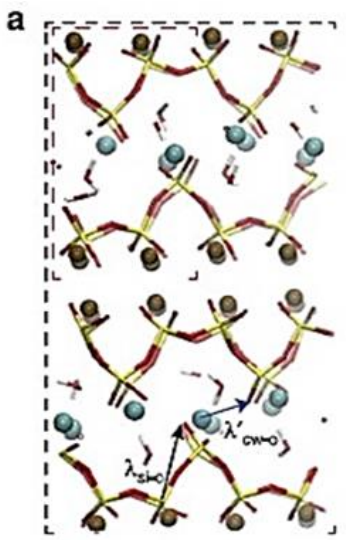

b<smiles>C=CC(C)(C)C(C)(C)C(C)(C)C1C(C)(C)CC1(C)C</smiles><smiles>CC(C)(C)C1(C)C2(C)CCC(C)(C2)C1(C)C</smiles>

Cadeia de Sílica

C

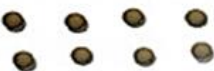

intercamada de Calcio

d

Q 0 \&

intracamada de Cálcio

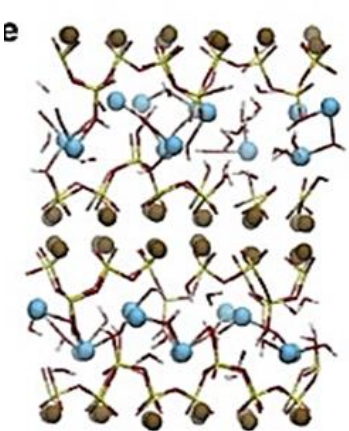

$\mathrm{Ca} / \mathrm{Si}=1.1$

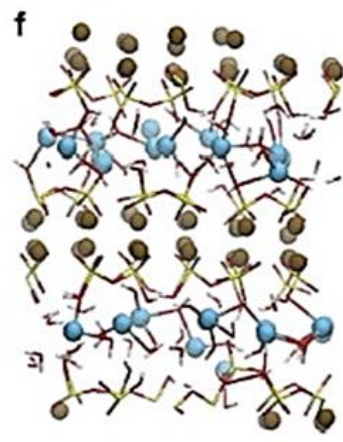

$\mathrm{Ca} / \mathrm{Si}=1.5$

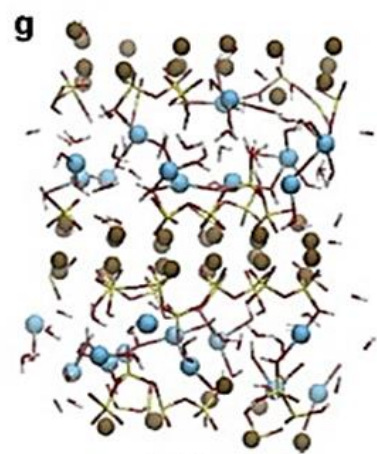

$\mathrm{Ca} / \mathrm{Si}=1.8$

Figura 7: C-S-H, Estrutura química, diferentes relações Ca/Si. Fonte: Adaptado de Abdolhosseini Qomi et al.

\subsection{Análise de MEG/FEG}

$\mathrm{Na}$ análise qualitativa por microscopia eletrônica de varredura (MEV/FEG), pode-se observar que tanto o traço R, figura 8-a, quanto o traço T2, figura 8-b, possuem matriz com presença expressiva de macroporos, inclusive alguns na ordem de $200 \mu \mathrm{m}$. Esta situação torna a matriz menos densa pela presença da alta porosidade resultando no aumento da permeabilidade, figura 5-a, como também na baixa resistência à tração, conforme ilustra figura 6 .

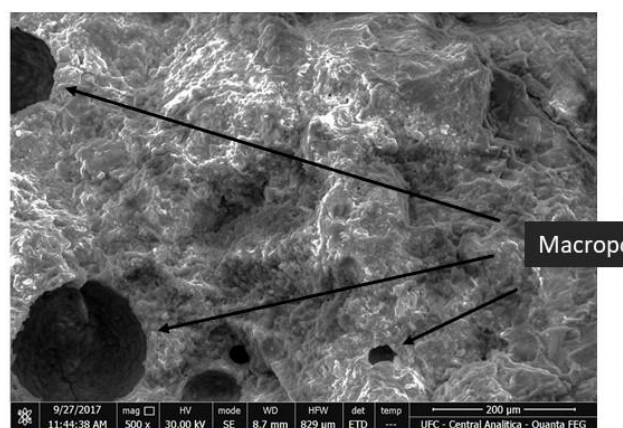

a)

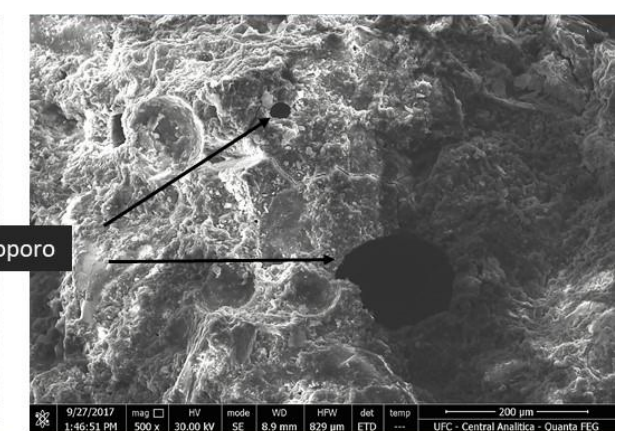

b)

Figura 08: Imagens MEV - a) traço R e b) T2. 
Os traços C2-1,2 e CT2-1,2, figuras 9-a e 9-b, respectivamente, apresentaram porosidade total reduzida assim como a quantidade total de macroporos. Os macroporos são de dimensões na ordem de $20 \mu \mathrm{m}$. O traço C2-1,2 apresenta mais falhas (microfissuras), figura 9-b e isto resultou na menor resistência à tração (16,5 $\mathrm{MPa})$ se comparado ao traço CT2-1,2 (17,1 MPa), conforme ilustra a figura 6.

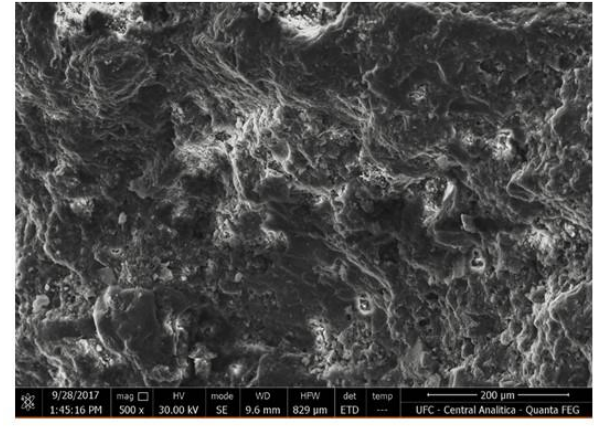

a)

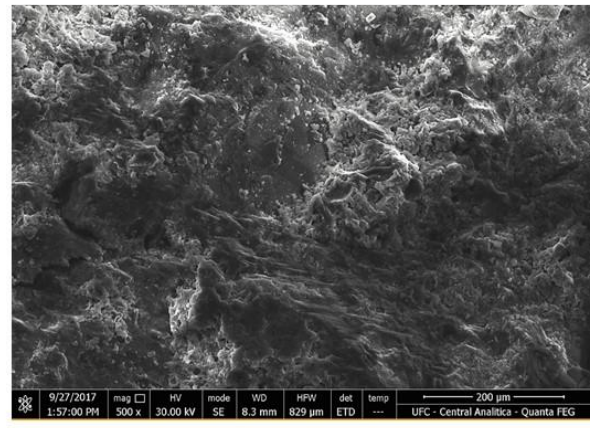

b)

Figura 9: Imagens MEV - a) traço C2-1,2 e b) CT2-1,2.

Os traços CT2-0,8 e C2-0,8, respectivamente, são menos porosos e a matriz é mais densa com pouca porosidade e poucas falhas perceptíveis, como ilustra a figura 10-a e 10-b.

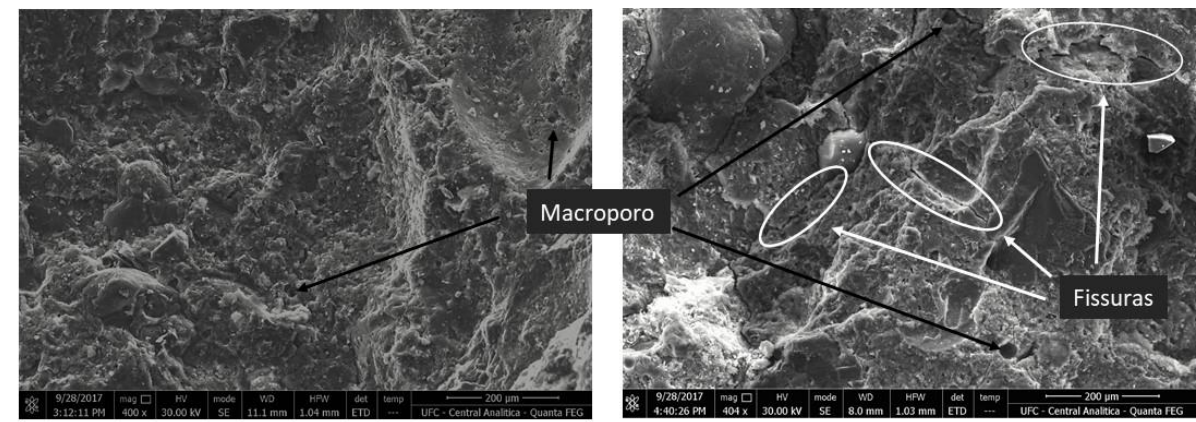

a)

Figura 10: Imagens MEV - traços a) CT2-0,8 e b) C2-0,8.

Outra observação é que na figura 11 , o traço CT2-0,8 apresenta crescimento de material cristalino no interior de alguns macroporos.

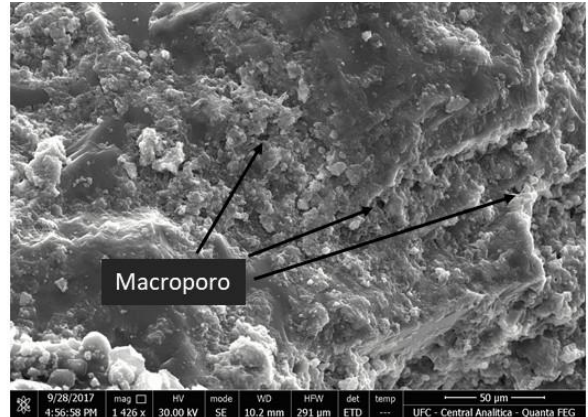

a)

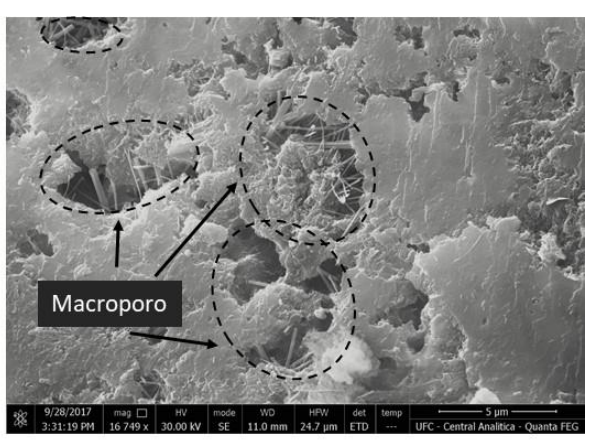

Figura 11: Imagens MEV - traços CT2-0,8.

Este fato é importante pois ocasiona a redução do tamanho dos poros o que pode até mudar sua classificação de macroporo para mesoporo ou ainda de mesoporo para um microporo.

Esta transformação das dimensões dos poros é o que se chama de refinamento dos poros, que provoca modificação da distribuição da porosidade. Embora a porosidade total possa não se modificar ou até mesmo 
aumentar, o refinamento é sempre positivo para redução da permeabilidade.

As adições empregadas resultaram na melhora da microestrutura dos compósitos pois serviram de núcleos para a formação de C-S-H secundário, em locais onde provavelmente apenas existiriam poros conforme ilustra figura 12.

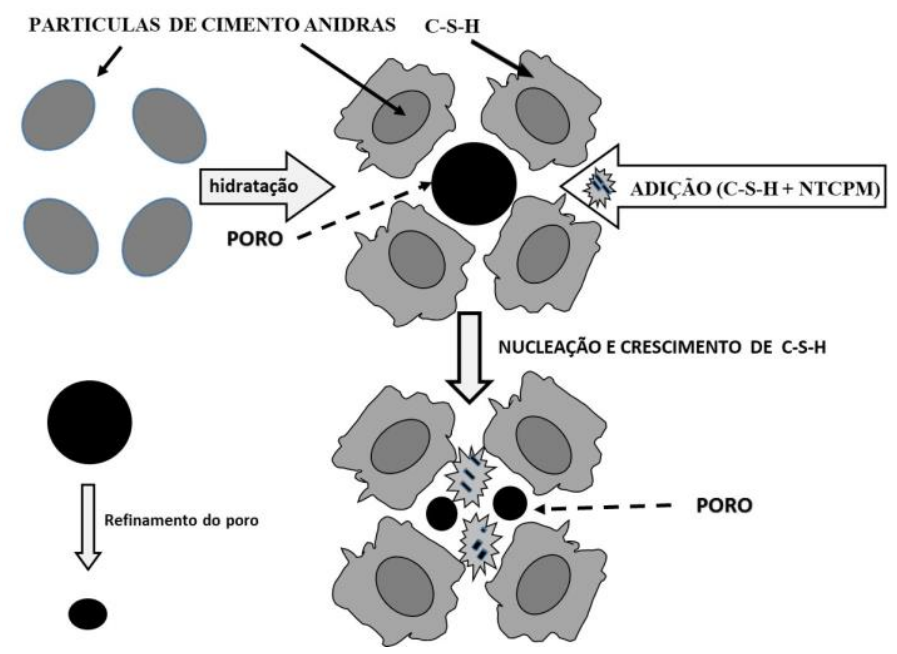

Figura 12: Esquema de nucleação e crescimento de partículas de C-S-H secundário.

As partículas de C-S-H (precipitadas) apresentam a mesma origem química do C-S-H formado pela hidratação dos silicatos do cimento Portland. Os traços com C-S-H com relação $\mathrm{Ca} / \mathrm{Si}=0,8$ apresentaram melhores resultados quando comparados aqueles traços com adição de $\mathrm{C}-\mathrm{S}-\mathrm{H}$ de relação $\mathrm{Ca} / \mathrm{Si}=1,2$.

As partículas de NTCPM usadas como adição isolada (sem C-S-H) parecem não ter resultado em melhoras microestruturais consideráveis, apenas um discreto aumento na resistência à tração (13\%), em relação ao traço referência, conforme figura 06, isto se deve pela alta resistência à tração deste tipo de partículas e que acabam resistindo ao início das primeiras microfissuras como um reforço microestrutural.

A adição de NTCPM combinada com as adições de C-S-H, em especial de relação $\mathrm{Ca} / \mathrm{Si}=0,8$, resulta em redução da porosidade total e distribuição dos poros mais refinada. Assim a adição combinada provoca redução da permeabilidade, aumento da densidade e ainda aumento considerável da resistência a tração, como foi o caso do traço CT2-0,8.

\section{CONCLUSÕES}

Foi possível através deste estudo, concluir que adições de C-S-H de relação $\mathrm{Ca} / \mathrm{Si}=0,8$ resultaram em compósitos com: melhores resultados de resistência à tração; menor porosidade total; distribuição dos poros mais refinada e maior densidade.

Os compósitos com NTCPM, traço T2, apresentou elevação discreta da resistência à tração quando comparadas ao traço referência, porém, considerando as características de porosidade total e distribuição dos poros, os resultados não foram relevantes em relação ao traço referência.

A combinação das adições de C-S-H e NTCPM, resultou nos compósitos com melhores características. Pode-se concluir que esta combinação usa o benefício da resistência a tração dos NTCPM também da reatividade química do $\mathrm{C}-\mathrm{S}-\mathrm{H}$ em especial com relação $\mathrm{Ca} / \mathrm{Si}=0,8$, que causa a nucleação e crescimento de materiais resistentes como o C-S-H secundário de estrutura química ordenada e sem falhas. O crescimento destes cristais nos espaços porosos pode melhorar a microestrutura tornando-a mais densa com porosidade refinada e ainda ocupa os espaços porosos evitando seu preenchimento por cristais de baixa resistências e eventualmente solúveis como o $\mathrm{Ca}(\mathrm{OH})_{2}$ que tende a ocupar em média $25 \%$ do volume de sólidos nos concretos convencionais. 


\section{BIBLIOGRAFIA}

[1] MEDEIROS, M.H.F. et al. Compósitos de cimento Portland com adição de nanotubos de carbono (NTC): Propriedades no estado fresco e resistência à compressão. Revista Matéria, Rio de Janeiro.v.20, n.1, pP.127144, 2015.

[2] VEEDU, V.P. Multifunctional cementitious nanocomposite material and methods of making the same. Patente: US 7666327 B1 (2010).

[3] THOMAS, J.J., JENNINGS, H.M., CHEN, J.J. Influence of nucleation seeding on the hydration. The Journal of Physical Chemistry, Washington, DC, v.113, pP.4327-4334, fev. 2009.

[4] KUNTHER, W., FERREIRO, S., SKIBSTED, J. Influence of the Ca/Si ratio on the compressive strength of cementitious calcium silicate hydrate binders. J. Mater. Chem. A, 2017, 5, 17401-17412. DOI: $10.1039 / \mathrm{c} 7 \mathrm{ta06104h}$.

[5] KIRGIZ, M.S. (2014). Effects of Blended Cement Paste Chemical Composition Changes on Some Strength Gains of Blended Mortars. The Scientific World Journal, 2014, 625350. Disponível em: <http://doi.org/10.1155/2014/625350>, acessado em 10 de novembro de 2018.

[6] TAYLOR, H.F.W. Proposed Structure for Calcium Silicate Hydrate Gel. J. Am. Ceram. Soc. N. 69, p. 464-467, 1986.

[7] TAYLOR, H.F.W. Cement Chemistry, Thomas Telford Publishing, 2.ed. 1997.

[8] DUART, M.A. Efeitos da adição de nanotubos de carbono e C-S-H precipitado nas propriedades de nanocompósitos cimentícios. 2017. 130 f. Tese (Doutorado), UFN, Santa Maria, 2017.

[9] MOKHTAR, A. et al. Functionalization and characterization of carbon nanotubes/polypropylene nanocomposite. World Academy of Science, Engineering and Technology. v.58, p. 421-425, 2011.

[10] ASSOCIAÇÃO BRASILEIRA DE NORMAS TÉCNICAS. NBR 16697: Cimento Portland - Requisitos. Rio de Janeiro, 2018.

[11] ASSOCIAÇÃO BRASILEIRA DE NORMAS TÉCNICAS. NBR 7214: Areia normal para ensaio de cimento - Especificação. Rio de Janeiro, 2015.

[12] ASSOCIAÇÃO BRASILEIRA DE NORMAS TÉCNICAS. NBR 13279: Argamassa para assentamento e revestimento de paredes e tetos - Determinação da resistência à tração na flexão e à compressão. Rio de Janeiro, 2005.

[13] IUPAC. Compendium of Chemical Terminology, 2nd ed. (the "Gold Book"). Compiled by A. D. McNaught and A. Wilkinson. Blackwell Scientific Publications, Oxford (2014). ISBN 0-9678550-9-8. Disponível em: https://doi.org/10.1351/goldbook. Acessado em: 10 mar. 2017.

[14] EVANGELISTA, A.C.J. et al. Evaluation of Carbon Nanotube Incorporation in Cementitious Composite Materials. Materials (Basel). 2019; 12. DOI:10.3390/ma12091504.

[15] ABDOLHOSSEINI QOMI, M.J. et al. Combinatorial molecular optimization of cement hydrates. Nature. Springer Nature. Nova Yorke. 2014.

\section{ORCID}

Marcelo Adriano Duart ～https://orcid.org/0000-0002-9256-5580 